\title{
Digital material laboratory: Wave propagation effects in open-cell aluminium foams
}

\author{
E.H. Saenger ${ }^{\mathrm{a}}$, D. Uribe ${ }^{\mathrm{b}, \mathrm{c}}$, R. Jänicke ${ }^{\mathrm{b}}$, O. Ruiz ${ }^{\mathrm{c}}$, H. Steeb ${ }^{\mathrm{b}}$ \\ ${ }^{a}$ Geological Institute, ETH Zürich, Switzerland \\ ${ }^{b}$ Institute of Mechanics, Ruhr-University Bochum, Germany \\ ${ }^{c} C A D / C A M / C A E$ Laboratory, EAFIT University, Medellin, Colombia
}

\begin{abstract}
This paper is concerned with numerical wave propagation effects in highly porous media using digitized images of aluminum foam. Starting point is a virtual material laboratory approach. The Aluminum foam microstructure is imaged by 3D X-ray tomography. Effective velocities for the fluid-saturated media are derived by dynamic wave propagation simulations. We apply a displacement-stress rotated staggered fnite-difference grid technique to solve the elastodynamic wave equation. The used setup is similar to laboratory ultrasound measurements and the computed results are in agreement with our experimental data. Theoretical investigations allow to quantify the influence of the interaction of foam and fluid during wave propagation. Together with simulations using an artificial dense foam we are able to determine the tortuosity of aluminum foam.
\end{abstract}

Keywords:

Computational material physics, Wave propagation, Finite-difference modeling, X-ray tomography, Aluminium foam

Email address: erik.saenger@ethz.ch ( E.H. Saenger) 


\section{Introduction}

Digital material methodology combines modern microscopic imaging with advanced numerical simulations of the physical properties of materials. One goal is to complement physical laboratory investigations for a deeper understanding of relevant physical processes. Large-scale numerical modeling of elastic wave propagation directly from the microstructure of the porous material is integral to this technology.

In this paper, we numerically consider a highly porous, open-cell aluminium foam. This special material is suitable for various applications in mechanics and engineering, e. g. as light-weight construction elements, mechanical filters or chemical catalysers. Besides its own applicability, open-cell aluminium foam has certain mechanical properties (porosity, intrinsic permeability, tortuosity etc.) which are similar to various cellular materials such as trabecular bone or polyurethane foam. Thus, various results of the present investigation can be transformed directly e. g. to the non-invasive diagnostics of cancellous bone.

Open-cell aluminium foam can be fabricated using open-cell polymer foam as template structure which is replaced by aluminium during a casting process. The resulting aluminium skeleton is built up as an irregular polyhedral network accounting for high porosity and effective hydraulic permeability, cf. Figure 1.

In order to investigate the complex wave propagation phenomena in this material, we split the considerations in three parts. First, we explain our applied digital material workflow. The specific workflow is put into context 
with other known approaches. Second, we discuss and present a specific numerical setup to investigate highly porous media using finite-difference wave simulations on a microscale. Third, we present and evaluate the results for aluminium foam. These numerical results, which contain the physical processes on the pore scale, allow us to understand observations on a much larger scale (i.e., the sample scale).

\section{Characterization of the material}

For the present study, we analyze a 10 ppi AlSi7Mg foam (m.pore GmbH, Dresden, Germany). For quasi-static loading conditions, this material exhibits a linear-elastic range followed by a pronounced plateau stress in the stress-strain relation (Gibson and Ashby, 1997; Nieh et al., 2000). Already for moderate compressive stresses, single layers of the skeleton start to fail which leads to a subsequent collapse of the entire structure. However, this paper will focus on sound and ultrasound propagation effects with small amplitudes and we restrict ourselves on purely elastic material properties. The bulk properties of aluminium forming the porous skeleton can be characterized with standard testing methods. The mechanical properties of the ligaments (Youngs modulus, Poissons ratio, density) are known from quasistatic experiments (Jang et al., 2008), cf. Table 1.

In Figure 2, a typical pore of the investigated foam is depicted. The morphological properties discussed in this section are derived from the depicted single cell, which we consider to be representative for all cells. Stochastical considerations are not included in this part of the paper. Due to gravity effects during the processing of the polymer template, an intrinsic anisotropy 
of the structure can be observed. The cells are elongated in $\mathrm{z}$ direction by the anisotropy factor $\tau=1.25$. Note that, within this contribution, all mechanical and numerical experiments are carried out with respect to this elongated $z$ direction. We measure the cell size to account $7.3 \mathrm{~mm}$ in $z$ direction and $5.8 \mathrm{~mm}$ in $x$ and $y$ direction, respectively. Furthermore, we find a typical ligament length of $2.0 \mathrm{~mm}$. In Figure 3, the cross sections of one ligament are depicted. Again, the depicted ligament is considered to be representative for all ligaments within the specimen without further stochastical considerations. One observes the ligament to take a bone shape with a cross section which varies from a nearly triangular shape close to the ligament nodes to a more and more circular shape with increasing distance from the nodes. At the central position we observe a cross section area of $\mathrm{A}=0.1912 \mathrm{~mm}^{2}$.

Altogether, we find the micromorphological properties to be close to the properties of the foam evaluated in Jang et al. (2008). However, the cross sectional areas of the ligaments given by Jang et al. $\left(\mathrm{A}=0.296 \mathrm{~mm}^{2}\right)$ seem to be considerably larger than for the microstructure investigated in the present contribution. Thus, the effective material properties, in particular the effective Young's modulus of the skeleton, can also be expected to be considerably lower than that one found by Jang et al. (2008).

Besides the material properties of the ligaments the effective properties of the skeleton are to be investigated in mechanical experiments. As data basis, a set of 3 aluminium foam samples $(40 \mathrm{~mm} \times 40 \mathrm{~mm} \times 40 \mathrm{~mm}$ ) has been investigated. The average porosity has been measured by weighing as $\phi=$ 0.933. By uniaxial compression tests (Schenck-Trebel universe testing device, $1 \mathrm{kN}$ load cell) in direction of elongated cells ( $z$ direction) within the linear- 
elastic (pre-buckling) regime have resulted in the Young's modulus of the frame $E=55.21 \mathrm{MPa}$ (standard deviation $5.30 \mathrm{MPa}$ ) and a bulk modulus of $K=18.40 \mathrm{MPa}$ (standard deviation 1.77 MPa). In the experiments, a Poisson effect could not be observed, i.e. we assume that Poisson's ratio equals zero $(\nu=0)$.

Let us compare our data first to the analytical estimations by Gibson and Ashby (1997), where Young's modulus of the frame is proposed to equal

$$
E=C_{1} E^{s}(1-\phi)^{2} .
$$

If the fitting parameter is, as usual in the related literature, assumed to $\mathrm{C}_{1}=1$, Gibson and Ashby predict $E=277.83 \mathrm{MPa}$. By contrast, the experimental data by Jang et al. (2008) determine $E=595.47 \mathrm{MPa}$, i. e. one order of magnitude larger than our measurement. However, we want to state that the results can not be expected to be identical. Whereas the analytic estimation by Gibson and Ashby assumes a simplified cell geometry, the measurements of Jang et al. are related to a skeleton with slightly different properties of the single cells. In particular the diameter of the ligament cross sections, which has been observed to be considerably larger in the frame investigated by Jang and al., influences considerably the effective properties for local bending dominated deformations during overall compression of the sample.

At that time, no further geometrical and hydro-mechanical properties such as the effective hydraulic permeability have been determined yet in physical experiments. 


\section{Digital material laboratory workflow}

3D X-ray microtomographic imaging and subsequent numerical determination of effective material properties is nowadays applied by various groups (申ren et al., 2007; Sakellariou et al., 2007; Malinouskaya et al., 2008; Dvorkin et al., 2008; Saenger et al., 2011), to name only a few. Tomographic imaging can be routinely performed over three orders of magnitude in length scale with correspondingly high data fidelity. This capability, coupled with the development of advanced computational algorithms for image interpretation, three-dimensional visualization, and structural characterization and computation of physical properties based on image data, allows for a numerical laboratory approach to study real heterogeneous materials (Sakellariou et al., 2007).

Several processing steps are important for such a virtual material laboratory. It starts with a Computer Tomographic (CT) scan of a selected material sample. The phase segmentation, which can be complicated for strongly heterogeneous materials, is required to build an appropriate digital model (Fredrich et al., 1995). This model can be used for flow simulations (Arns et al., 2001; Keehm et al., 2004; Harting et al., 2005). They can provide realistic distributions of multiple fluids (e.g., patterns of wetting and nonwetting fluids) and effective transport properties (e.g., permeability). With exactly the same digital rock structure effective mechanical properties can be defined (Arns et al., 2002; Saenger, 2008; Madadi et al., 2009). This allows to consider relationships between transport and mechanical properties, both depending on the microscopical properties.

At this point, it is also possible to calibrate the numerical results with 
laboratory measurements determining macroscopical effective properties (or vice versa). While the numerically determined permeability values fit the experimental data relatively well, there is a mismatch for the mechanical properties in the case this technique is applied to low porous rocks (Knackstedt et al., 2009; Zhang, 2010; Madonna and Saenger, 2011). A systematic numerical overestimation of elastic moduli is reported. A possible reason is the limited resolution of X-ray techniques; features below that limit seem to play an important role (e.g., physics of grain contacts and tiny unresolved voids or cracks). However, for the considered aluminium foam we do not expect such resolution issues. A next step is to go from static to dynamic wave propagation simulations (Saenger et al., 2005). Although the macroscopic theory of wave propagation in porous fluid-saturated media has been established 50 years ago (Biot, 1956) there are still many unresolved questions about the physical origin of attenuation, dispersion and high frequency wave propagation in such media.

We concentrate on the Rotated Staggered Grid (RSG) Finite-Difference (FD) method (Saenger et al., 2000) for pore-scale simulation of wave propagation in digitized materials.

\subsection{Digital aluminium Foam Sample}

X-ray-based tomographic microscopy (Marone et al., 2009) is suitable to provide data on the real pore microstructure of materials. To demonstrate the proposed workflow we have selected the open-cell aluminium foam introduced earlier. However, other samples can be used as well. In Figure 5 we illustrate our final digitized model after several processing steps. These include discontinuity detection, thresholding and region processing (Gonzales 
and Woods, 2002). The digital aluminium foam contains 2 different phases: solid aluminium phase (colored) and the porous space (transparent). For the numerical analysis, we use a 3D-image of the aluminium foam discretized on a regular cartesian grid with $400 \times 400 \times 400$ grid points. The pores as well as the aluminium are $100 \%$ connected besides some ligaments at the boundary.

\subsection{Numerical Simulation of Wave Propagation in Aluminium Foam}

To study wave propagation effects in the digitized aluminium foam sample (Figure 4) numerically, we use a technique similar to the approach described in (Saenger et al., 2004, 2011). The basic idea is to study speeds of elastic waves through heterogeneous materials in the long wavelength limit (pore size « wavelength) using the RSG FD algorithm (Saenger et al., 2000). In the case of fluid-saturated highly porous media (porosity $\geq 80 \%$ ) it is necessary to modify the numerical setup compared to the case for low porosity materials (porosity $\leq 35 \%$ ). This is described below. A review of related methods is given in (Saenger, 2008; Saenger et al., 2011).

For the purpose of studying wave propagation effects of aluminium foam the digitized material is embedded into a homogeneous region, Figure 5. In this study we assign to this region the elastic properties of non-viscous water. Related to a classical experimental setup, this is similar to throughtransmission-experiments in an immersion technique. This is in contrast to a homogeneous elastic stiff rock embedding applied in (Saenger et al., 2004). With this modified embedding we reach a better coupling to the saturated aluminium foam and a longer wavelength can be numerically inserted.

The full synthetic models are made up of $804 \times 400 \times 400$ grid points. We 
perform our experiments with periodic boundary conditions in the directions parallel to the wave propagation process. A body force plane source at the top of the model is applied. A plane $P$-wave generated in this way propagates through the numerical model as shown in Figure 6. The broadband source in our experiments is always the first derivative of a Gaussian wavelet. The dominant frequency is given among other modeling parameters in Table 1. All computations are performed with second order spatial FD operators and with a second order time update. With two planes of receivers at the top and at the bottom of the model, it is possible to measure the time-delay of the peak amplitude of the mean plane wave caused by the inhomogeneous structure of the digitized material sample. With the time-delay (compared to a reference model) one can estimate the effective velocity of compressional waves (Saenger et al., 2004). In general, two different kinds of P-waves can be expected for porous media. Fist a fast compressional wave and second a so-called slow (Biot) wave, cf. (Biot, 1956; Mavko et al., 2003).

Next, we analyze 2 distinct cases of fluid-saturated aluminium foam numerically. At first, we compute aluminium foam which is saturated with a (non-viscous) fluid. In the second case, we modify the properties of aluminium (Young's/compression modulus, density: factor 10 higher, each). Assuming Poiseuille flow we can calculate the viscous skin depth as a function of frequency $f$ (Hughes et al., 2003; Steeb, 2010)

$$
d=\sqrt{\frac{2 \eta^{f R}}{2 \pi f \rho^{f R}}},
$$

where $\rho^{f R}$ denotes the effective (true) density of the fluid and $\eta^{f R}$ the effective dynamic fluid viscosity. 
If the pore radius $r$ is larger than the viscous skin depth $d$ we investigate the high-frequency domain which is characterized by an additional inertia coupling mechanism between the solid and the fluid phase. As in our microstructure-based analysis the pore radius $r$ is known, we are able to calculate this critical frequency (transission frequency)

$$
f^{c r i t}=\frac{\eta^{f R}}{\rho^{f R} \pi r^{2}}<1 \mathrm{~Hz} .
$$

Even if we assume Johnson and Plona's formula (Johnson and Plona, 1982) to estimate a travelling second wave, known as the viscous frequency,

$$
f^{\text {iisous }}=\frac{\eta^{f R}}{\rho^{f R} \pi r^{2} \xi^{2}}, \quad \xi \approx 0.01,
$$

we expect to end up in the high-frequency range if we apply frequencies above $100 \mathrm{~Hz}$.

\subsection{Case 1: Water-saturated Aluminuim Foam}

From a theoretical point of view we consider here the high frequency range of Biot's velocity relations because we saturate our digitized image of aluminium foam with a non-viscous fluid (effective dynamic fluid viscosity $\eta^{f R}=0$ ). In the case of highly porous media, the most dominant wave is that one which is travelling mainly through the fluid phase. The effective velocity is $v_{p, f a s t}=1487 \mathrm{~m} / \mathrm{s}$. This is slightly faster compared to the case of homogeneous water $\left(v_{p, \text { water }}=1480 \mathrm{~m} / \mathrm{s}\right)$. A coherent $P$-wave travelling through the aluminim skeleton can not be detected clearly. Only some incoherent low-amplitude wave arrivals are visible.

Similar results are obtained in ultrasound through-transmission laboratory experiments. At room temperature $\left(\Theta \approx 20^{\circ} C\right)$, we obtain a wave 
velocity in the water phase of $v_{p, w a t e r}=1498 \mathrm{~m} / \mathrm{s}$. If we perform an ultrasound experiment with the water-saturated aluminium foam sample, we observe a slightly faster $P$-wave of $v_{p, f a s t}=1512 \mathrm{~m} / \mathrm{s}$.

The numerical and experimental investigations can be compared to the high-frequency limits of Biot's equations (Biot, 1956; Mavko et al., 2003). In Figure 7 we show the solution of the wave velocity for the fast wave of Case 1. If the tortuosity is small, i.e. if $\alpha_{\infty} \approx 1$, the wave velocity is slightly larger $v_{p, \text { fast }}=1507 \mathrm{~m} / \mathrm{s}$ than the $P$-wave travelling through water $\left(v_{p, \text { water }}=1483\right.$ $\mathrm{m} / \mathrm{s}$ ). For larger values of tortuosity, the wave velocity becomes smaller. Note that we obtain a wave speed $v_{p, f a s t}=1487 \mathrm{~m} / \mathrm{s}$ in case of $\alpha_{\infty}=1.14$.

\subsection{Case 2: Water-saturated Artificial Stiff Aluminium Foam}

For this case, we assign to the aluminium foam stiffness and density parameters which are ten times higher then in Case 1. With this trick, from a numerical point of view very straightforward, we have an artificial stiff alu-

minium foam. With this simulation we want to understand if a signature of the elastic properties of the foam will influence the speed of the fast $P$-wave. Furthermore, we are able to examine the amount of tortuosity from this setup. From our numerical experiment we obtain in that case a fast $P$-wave velocity of $v_{p, f a s t}=1409 \mathrm{~m} / \mathrm{s}$. We therefore conclude that the measured wave speed for Case 1 is not a result of the fluid properties only. Comparing the numerical investigations with the high-frequency limits of Biot's equations, we are able to determine the turtuosity parameter of our aluminium foam. As turtuosity is the only physical effect which is responsible for a deviation of the fast $P$-wave from the wave travelling through homogeneous water, we obtain for the foam $\left(v_{p, f a s t}=1409 \mathrm{~m} / \mathrm{s}\right)$ the turtuosity $\alpha_{\infty}=1.14$. 
Evaluating Biot's equation for Case 1 with $\alpha_{\infty}=1.14$ predicts the theoretical P-wave velocity of water-saturated aluminium foam of $v_{p, f a s t}=$ $1487 \mathrm{~m} / \mathrm{s}$ which is exactly the velocity we have observed in our numerical simulation.

\section{Conclusions}

In this paper we describe numerical, laboratory and theoretical estimations of effective acoustic properties of a highly porous media. The considered aluminum foam with a porosity of 0.923 is imaged by 3D X-ray tomography. With the known material properties of Aluminum and water we perform large-scale finite-difference wave propagation computations to estimate wave velocities of the fully saturated foam. Due to the high porosity we have to apply a specific numerical setup where the saturated sample is inserted in a homogeneous fluid environment. The laboratory and numerical measurements show excellent agreement. A detailed theoretical analysis, supported with simulations using artificial dense aluminum, gives three main results: First, our experiments took always place in the high-frequency limit of the Biot-theory. Second, the interaction between foam and fluid can not be neglected for estimating wave propagation effects. Third, we are able to determine the tortuosity of the aluminum foam to equal $\alpha_{\infty}=1.14$.

[1] Arns, C. H., Knackstedt, M. A., Pinczewski, W. V., 2001. Accurate estimation of transport properties from microtomographic images. Geophys. Res. Lett. 28, 33613364.

[2] Arns, C. H., Knackstedt, M. A., Pinczewski, W. V., Garboczi, E. J., 2002. Computation of linear elastic properties from microtomographic 
images: Methodology and agreement between theory and experiment. Geophysics 67, 13961405.

[3] Biot, M. A., 1956. Theory of propagation of elastic waves in a fluidsaturated porous solid I. Low-frequency range. J. Acoust. Soc. Amer. 29, 168191.

[4] Dvorkin, J., Armbruster, M., Baldwin, C., Fiang, Q., Derzhi, N., Gomez, C., Nur, B., Nur, A., 2008. The future of rock physics: computational methods vs. lab testing. First Break 26, 6368.

[5] Fredrich, J., Menendez, B., Wong, T., 1995. Imaging the pore structure of geomaterials. Science 268(5208), 276279.

[6] Gibson, L. J., Ashby, M. F., 1997. Cellular solids. Structure and properties. Cambridge Solid State Science Series. Cambridge University Press, Cambridge.

[7] Gonzales, R. C., Woods, R. E., 2002. Digital Image Processing. PrenticeHall.

[8] Harting, J., Chin, J., Venturoli, M., Coveney, P. V., 2005. Large-scale lattice boltzmann simulations of complex fluids: advances through the advent of computational grids. Philos. T. R. Soc. A 363, 18951915.

[9] Hughes, E. R., Leighton, T. G., Petley, G. W., White, P. R., Chivers, R. C., 2003. Estimation of critical and viscous frequencies for biot theory in cancellous bone. Ultrasonics 41, 365368. 
[10] Jang, W.-Y., Kraynik, A. M., Kyriakides, S., 2008. On the microstructure of open-cell faoms and its e.ect on elastic properties. Int. J. Solids Struct. $45,18451875$.

[11] Johnson, D. L., Plona, T. J., 1982. Acoustic slow waves and the consolidation transition. J. Acoust. Soc. Am. 72, 556565.

[12] Keehm, Y., Mukerji, T., Nur, A., 2004. Permeability prediction from thin sections: 3D reconstruction and lattice-boltzmann flow simulation. Geophys. Res. Lett. 31, L04606.

[13] Knackstedt, M. A., Latham, S., Madadi, M., Sheppard, A., Varslot, T., Arns, C. H., 2009. Digital rock physics: 3D imaging of core material and correlations to acoustic and flow properties. The Leading Edge 28(1), 28 33.

[14] Madadi, M., Jones, A. C., Arns, C. H., Knackstedt, M. A., 2009. 3D imaging and simulation of elastic properties of porous materials. Comput. Sci. Eng. 11(4), 6573.

[15] Madonna, C., Saenger, E. H., 2011. Digital rock physics: Calibration with laboratory measurements. 73nd EAGE Conference, Vienna, Austria.

[16] Malinouskaya, I., Mourzenko, V.V., Thovert, J.-F., Adler, P. M., 2008. Wave propagation through saturated porous media. Phys. Rev. E 77, 066302.

[17] Marone, F., Hintermuller, C., McDonald, S., Abela, R., Mikuljan, G., Isenegger, A., Stampanoni, M., 2009. X-ray Tomographic Microscopy at 
TOMCAT. Journal of Physics: Conference Series 168, doi:10.1088/1742 6596/186/1/012042. 
[18] Mavko, G., Mukerji, T., Dvorkin, J., 2003. The Rock Physics Handbook. Tools for seismic analysis in porous media. Cambridge University Press, Cambridge.

[19] Nieh, T. G., Higashi, K., Wadsworth, J., 2000. E.ect of cell morphology on the compressive properties of open-cell aluminium foams. Mat. Sci. Eng. A 283, 105110.

[20] Фren, P.-E., Bakke, S., Held, R., 2007. Direct pore-scale computation of material and transport properties for north sea reservoir rocks. Water Resour. Res. 43, W12S04.

[21] Saenger, E. H., 2008. Numerical methods to determine effective elastic properties. Int. J. Eng. Sci., 598605.

[22] Saenger, E. H., Enzmann, F., Keehm, Y., Steeb, H., 2011. Digital rock physics: Effect of fluid viscosity on effective elastic properties. J. Appl. Geophys. 74, 236241.

[23] Saenger, E. H., Gold, N., Shapiro, S. A., 2000. Modeling the propagation of elastic waves using a modified finite-difference grid. Wave Motion 31(1), 77-92.

[24] Saenger, E. H., Krüger, O. S., Shapiro, S. A., 2004. Effective elastic properties of randomly fractured soils: 3D numerical experiments. Geophys. Prospect. 52(3), 183195.

[25] Saenger, E. H., Shapiro, S. A., Keehm, Y., 2005. Seismic effects of viscous Biot-coupling: Finite difference simulations on micro-scale. Geophys. Res. Letters 32, L14310. 
[26] Sakellariou, A., Arns, C. H., Sheppard, A. P., Sok, R. M., Averdunk, H., Limaye, A., Jones, A. C., Senden, T. J., Knackstedt, M. A., 2007. Developing a virtual materials laboratory. Mater. Today 10, 4451.

[27] Steeb, H., 2010. Ultrasound propagation in cancellous bone. Arch. Appl. Mech. 80, 489502.

[28] Zhang, Y., 2010. Modeling of the effects of wave-induced fluid motion on seismic velocity and attenuation in porous rocks (Ph.D. thesis). Massachusetts Institute of Technology. 
Used modeling parameters

Young's modulus of aluminium

$E^{s}=70.0 \mathrm{GPa}$

Poisson number of aluminium

$\nu^{s}=0.33$

Effective (true) density of aluminium

$\rho^{s R}=2700 \mathrm{~kg} / \mathrm{m}^{3}$

Bulk modulus of pore fluid

$K^{f}=1.48 \mathrm{GPa}$

Effective (true) density of pore fluid

$\rho^{f R}=1000 \mathrm{~kg} / \mathrm{m}^{3}$

Sample size (grid points)

$400 \times 400 \times 400$

Grid spacing

$\Delta h=60.331 \mu \mathrm{m}$

Sample thickness

$d=400 \times 60.331 \mu \mathrm{m}=0.0241 \mathrm{~m}$

Porosity

$\phi=0.923$ (from CT data)

Pore geometry

see Figure 2

Dominant frequency of used wavelet

$f_{\text {dom }}=24 \mathrm{kHz}$

Dominant wavelength@1480m/s

$\lambda_{\text {dom }}=0.061 \mathrm{~m} \approx 2.6 \mathrm{~d}$

Table 1: Modeling parameters and numerically estimated properties of the digitized aluminium sample shown in Figures 5. 
Experimentally obtained data

Porosity

$\phi=0.933$

Effective Young's modulus (skeleton)

$E=55.21 \pm 5.30 \mathrm{MPa}$

Effective Poisson's ratio (skeleton)

$\nu=0$

P-wave velocity (homogeneous water, $\Theta \approx 20^{\circ}$ )

$v_{p, w a t e r}=1498 \mathrm{~m} / \mathrm{s}$

P-wave velocity (water-saturated foam, $\Theta \approx 20^{\circ}$ ) $\quad v_{p, \text { fast }}=1512 \mathrm{~m} / \mathrm{s}$

Table 2: Experimental data of the aluminium foam samples. 


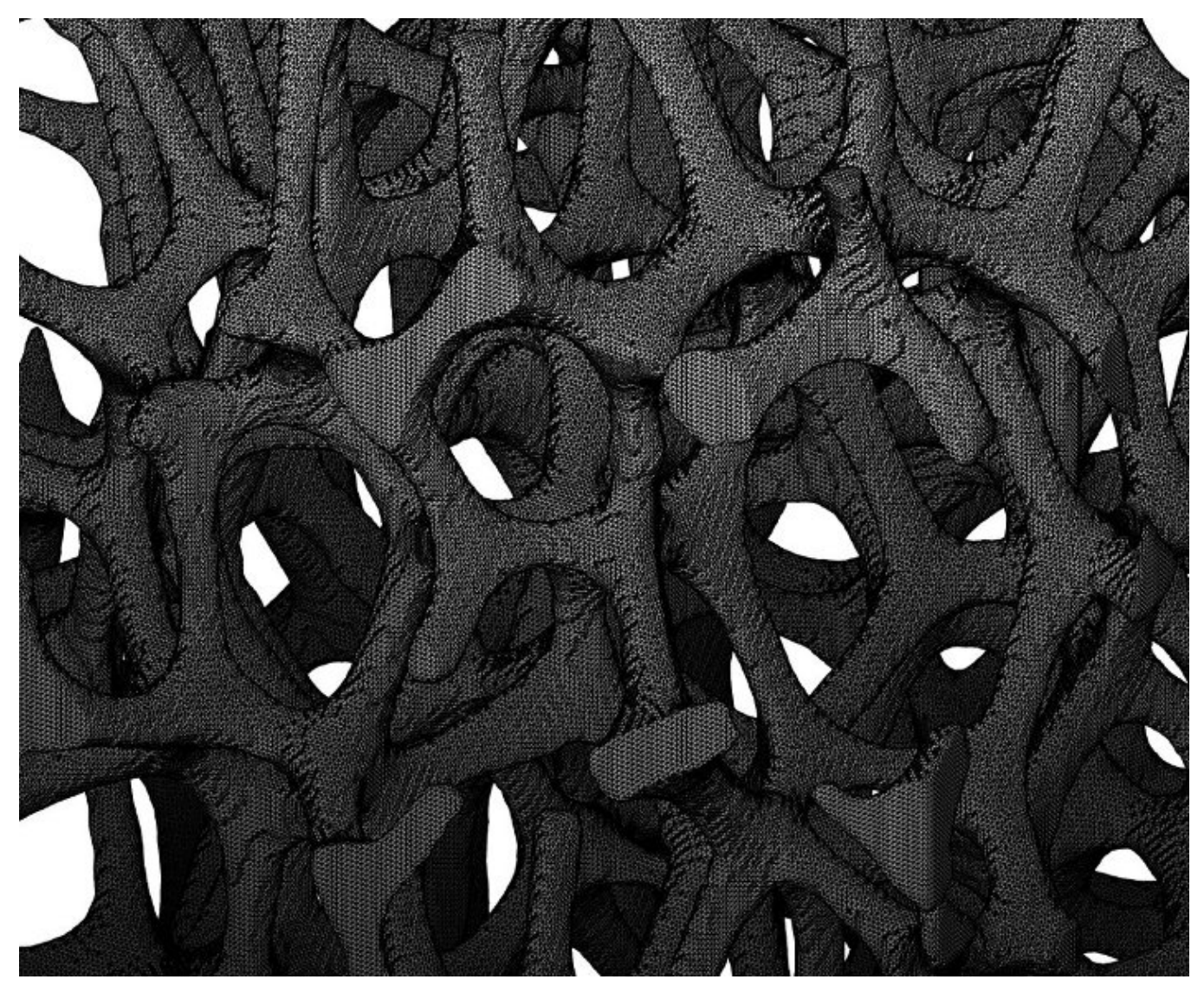

Figure 1: Irregular polyhedral network of the investigated open-cell aluminium foam. Detail from a CT reconstruction. 


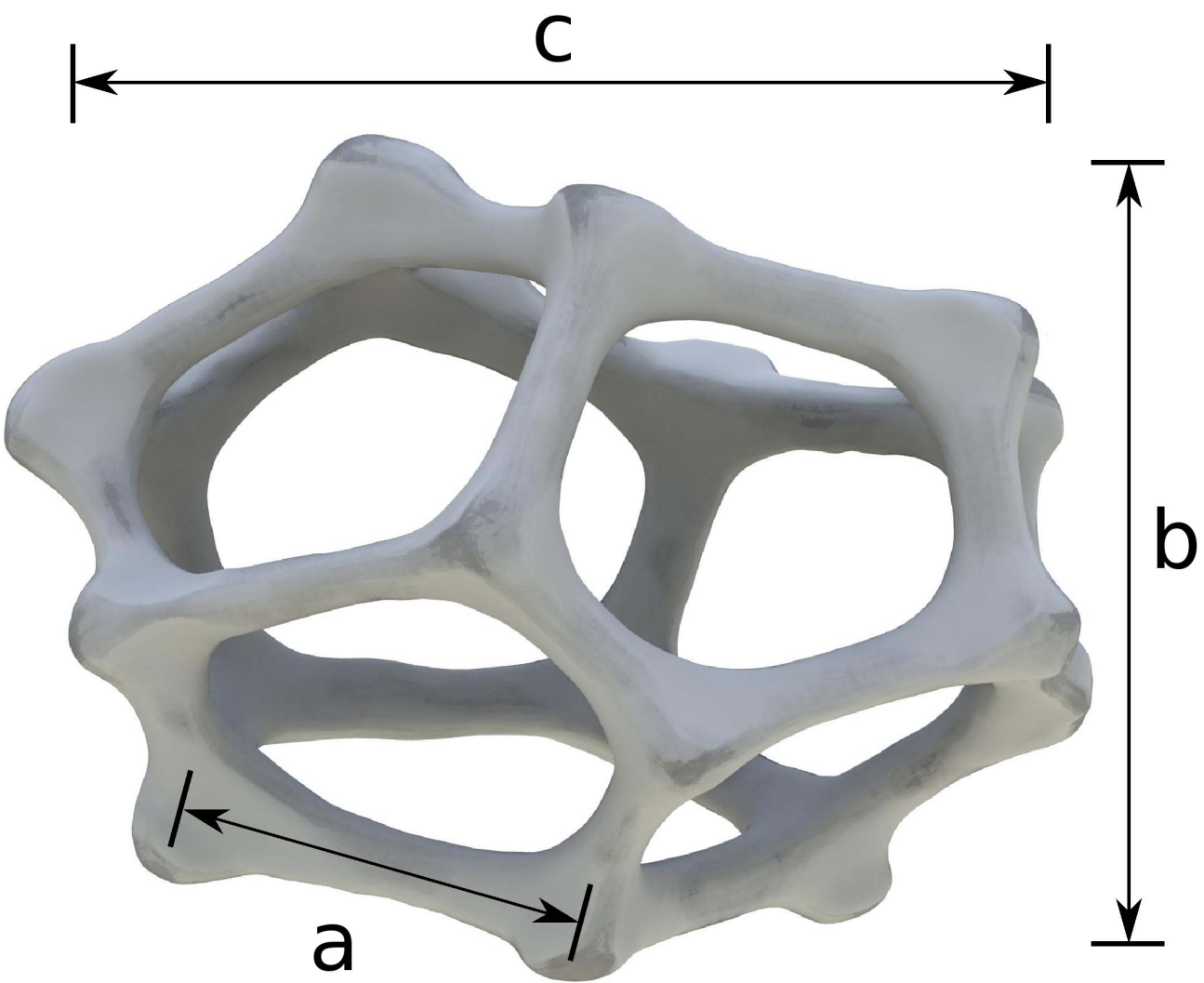

Figure 2: Exemplary cell of the aluminium foam with characteristic dimensions $a=$ $1.984 \mathrm{~mm}, b=5.836 \mathrm{~mm}, c=7.314 \mathrm{~mm}$ and anisotropy factor $\tau=c / b=1.25$. 


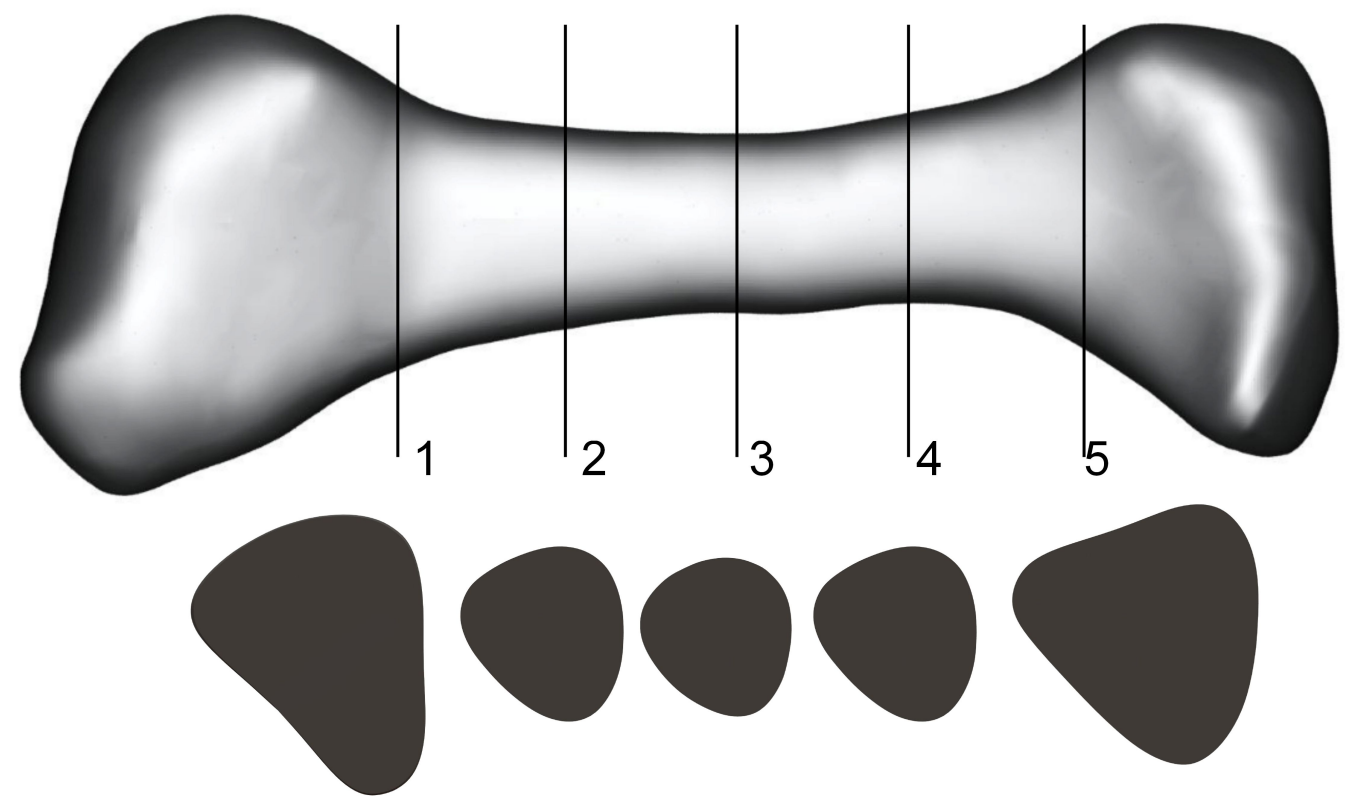

Figure 3: Single ligament of the aluminium foam with cross section areas $A_{1}=0.4616 \mathrm{~mm}^{2}$, $A_{2}=0.2081 \mathrm{~mm}^{2}, A_{3}=0.1913 \mathrm{~mm}^{2}, A_{4}=0.2289 \mathrm{~mm}^{2}$ and $A_{5}=0.4674 \mathrm{~mm}^{2}$. The cross section varies from a nearly triangular shape at the ligament nodes to a more circular shape in the ligament center. 


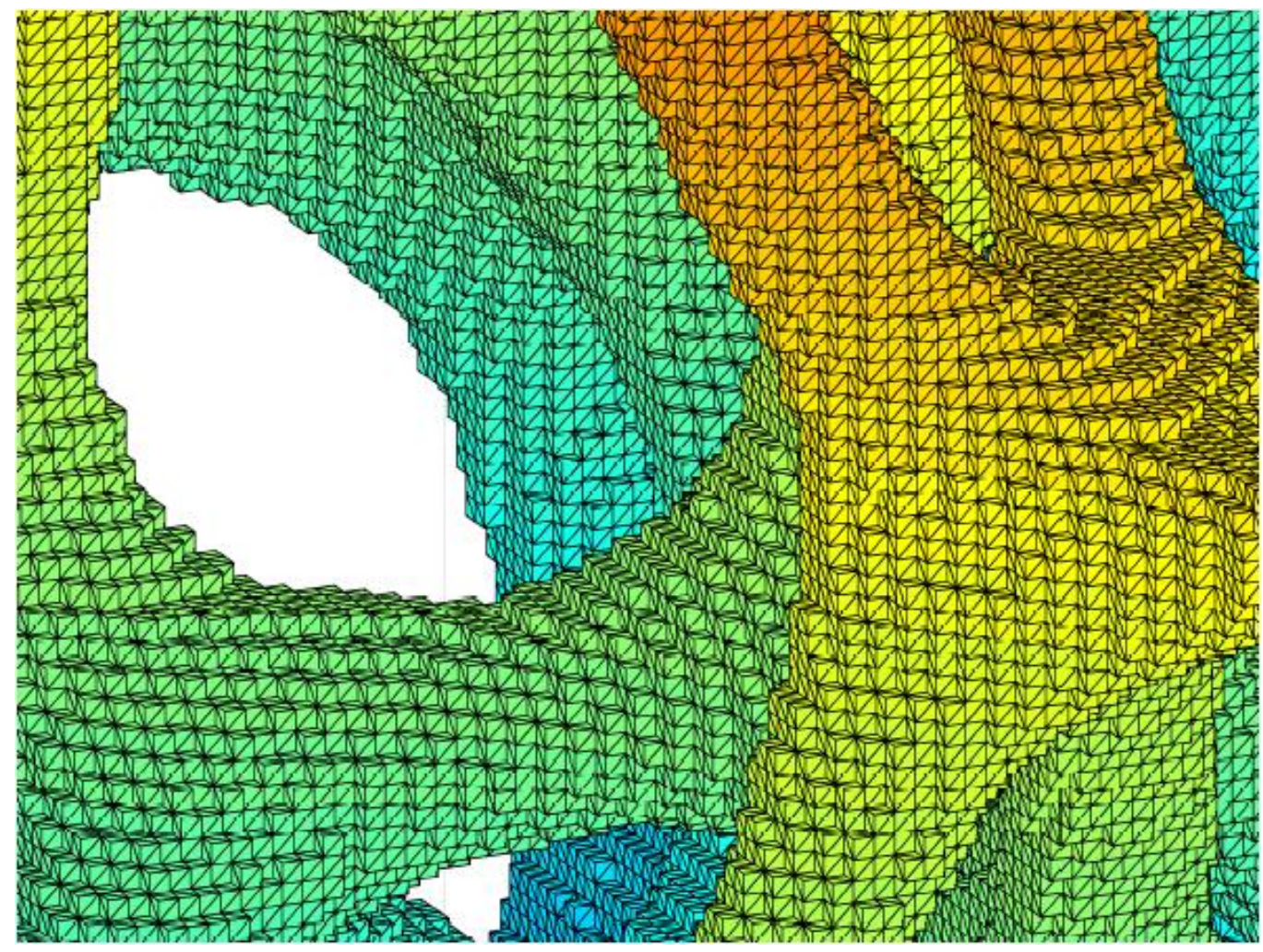

Figure 4: Voxel-based detail of the digitized aluminium foam model. The grid spacing accounts for $\Delta \mathrm{h}=60.331 \mu \mathrm{m}$. 


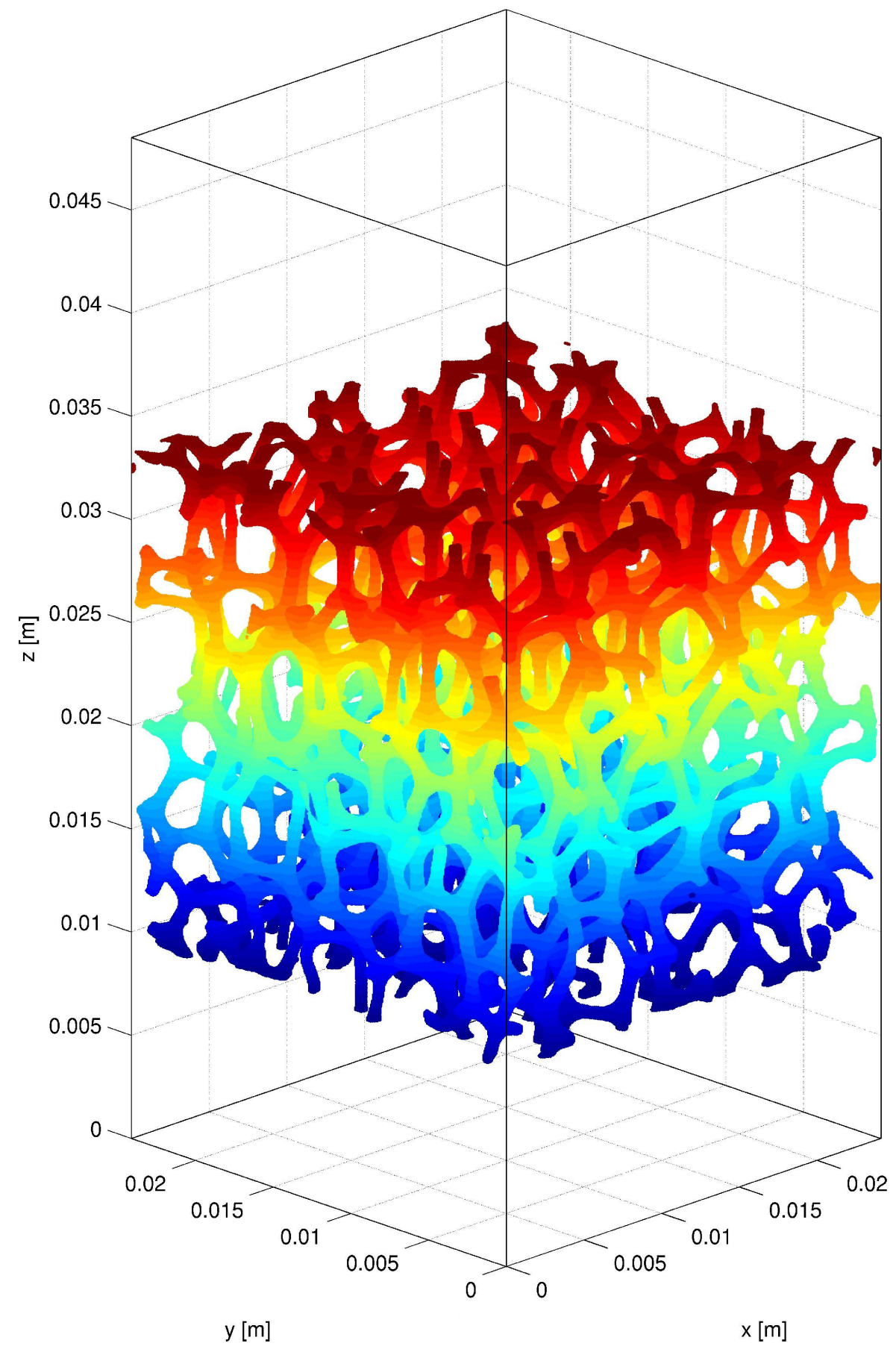

Figure 5: Investigated unit cell, aluminium (colored) and water (transparent). 


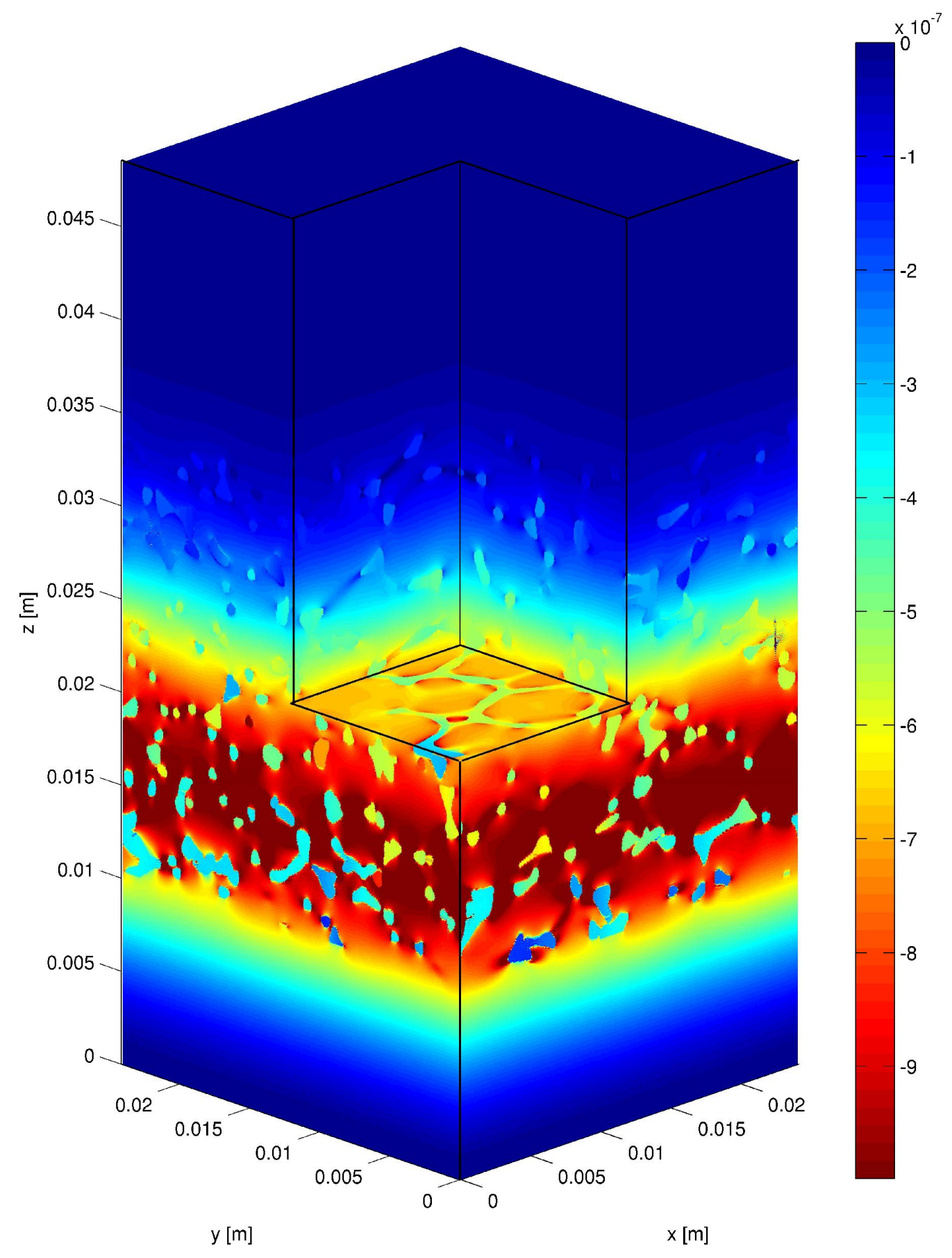

Figure 6: P-wave passing through the fluid-filled open-cell foam. 


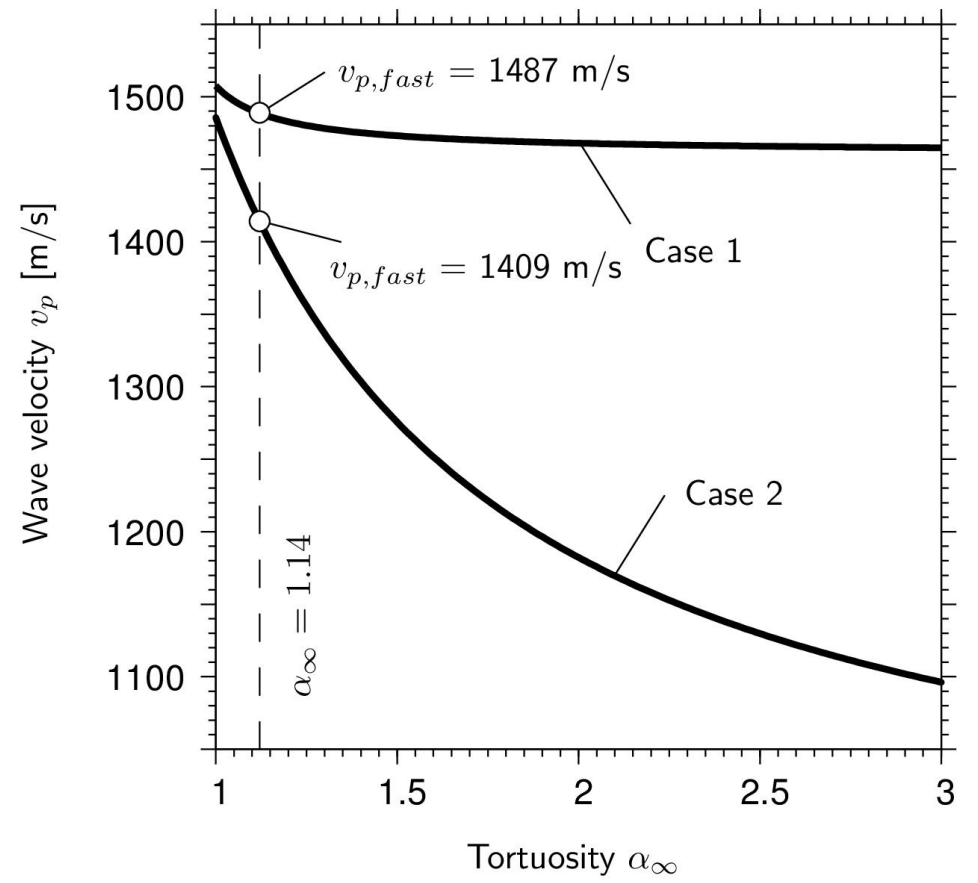

Figure 7: High-frequency solution of Biot's equations (Mavko et al., 2003). The phase velocity of the wave which mainly travels through the water phase is depicted for Case 1 and Case 2 depending on tortuosity. 